\title{
Parâmetros hematológicos e perfil bioquímico renal de cordeiros nascidos a termo e prematuros
}

\author{
[Hematologic parameters and renal biochemical profile in full-term and premature lambs]
}

\author{
F.L. F. Feitosa, J.F. Alcindo*, L.G. Narciso, F. Bovino, N.C. de Souza, L.C.N. Mendes,
} J.R. Peiró, S.H.V. Perri, L.G. Avila

\author{
Faculdade de Medicina Veterinária de Araçatuba - Unesp - Araçatuba, SP
}

\section{RESUMO}

O objetivo do presente estudo foi avaliar as variáveis hematológicas e o perfil bioquímico renal sérico de cordeiros nascidos a termo e prematuros do nascimento às 48 horas de vida, bem como verificar a influência da dexametasona sobre tais variáveis. Foram constituídos quatros grupos experimentais: PN (cordeiros nascidos de parto normal, $\mathrm{n}=15$, média de 146 dias); PNDEX (cordeiros nascidos de parto normal cujas mães receberam $16 \mathrm{mg}$ de dexametasona aos 141 de gestação, $\mathrm{n}=8$, média de 143 dias); PRE (cordeiros prematuros nascidos de cesarianas aos 138 dias de gestação, $\mathrm{n}=10$ ) e PREDEX (cordeiros prematuros nascidos de cesarianas aos 138 dias de gestação cujas mães receberam $16 \mathrm{mg}$ de dexametasona dois dias antes, $\mathrm{n}=9$ ). Os valores médios do volume globular e de hemoglobina diminuíram ao longo das 48 horas de observação, nos quatro grupos experimentais, porém dentro dos limites fisiológicos para a espécie. Houve variação da concentração plasmática de proteínas totais em todos os momentos, sendo os menores valores no grupo PRE. A contagem leucocitária foi mais alta no grupo PN apenas no M24h. Ao longo do período, apenas o grupo PN mostrou diferença entre o M24h e os demais momentos, e o grupo PRE apresentou os menores valores de neutrófilos no M0h, M15min e M60min. As concentrações séricas de creatinina foram mais altas no grupo PRE no M60min, M24h e M48h. Em todos os grupos, houve diminuição no M24h e M48h. Os parâmetros avaliados foram afetados pela prematuridade na espécie ovina e a dexametasona teve influência positiva sobre a taxa de sobrevivência dos animais prematuros.

Palavras-chave: ovinos, bioquímico, hematologia, prematuros

\begin{abstract}
The aim of the study was to evaluate hematologic parameters and renal biochemical profile of full-term and premature lambs from birth to 48 hours of life, and assess the effect of dexamethasone on such variables. Four experimental groups were formed: NDG (normal delivery group - lambs vaginally delivered, $n=15$, average of 146-day gestation); NDEXG (normal delivery with dexamethasone group - lambs vaginally delivered whose mothers received $16 \mathrm{mg}$ of dexamethasone at 141 days of gestation, $n=8$, average of 143-day gestation); PRE (premature lambs born by cesarean section at 138 days of gestation, $n=10$ ) and PREDEX (premature lambs born by cesarean section at 138 days gestation, whose mothers received $16 \mathrm{mg}$ of dexamethasone two days before, $n=9$ ). Mean values of cell volume and hemoglobin content decreased during the observation period of 48 hours in all groups, but within the physiologic limits for ovine species. There was significant variation in plasma concentration of total protein in all periods evaluated, with the lowest mean values in PRE group. The leukocyte count was significantly higher in PN group only in M24h. Throughout the observation period, only PN group showed differences between M24h and the other moments and PRE group showed the lowest values of neutrophils in M0h, M15min and M60min. Serum creatinine concentration was significantly higher in group PRE in M60min, M24h and M48h. In all groups, there was a decrease in M24 and M48. The evaluated parameters were affected by prematurity in sheep and dexamethasone had a positive effect on the survival rate of premature animals.
\end{abstract}

Keywords: sheep, biochemist, hematology, premature

Recebido em 1 de julho de 2016

Aceito em 29 de setembro de 2016

*Autor para correspondência (corresponding author)

E-mail: jefferson.alcindo@yahoo.com.br 


\section{INTRODUÇÃO}

O parto laborioso ou distócico pode levar ao comprometimento do feto e/ou da mãe. A literatura descreve que o método de nascimento pode modificar o equilíbrio imunológico no recém-nascido (Probo et al., 2012). O processo laborioso induz à leucocitose seletiva para neutrófilos e monócitos, com diferenças conforme o tipo de parto (Gasparoni et al., 1992; Herson et al., 1992; Thilaganathan et al., 1994).

As elevadas contagens totais de leucócitos em recém-nascidos a termo foram associadas a quadros de acidose respiratória e metabólica (Redüko et al., 2005). Bezerros recém-nascidos de cesarianas tendem a ser mais predispostos ao desenvolvimento da síndrome do desconforto respiratório e consequente acidose respiratória nas primeiras horas de vida, diferentemente do que ocorre na espécie humana (Cambier et al., 2000).

A asfixia, processo multifatorial que se desenvolve quando a oxigenação tecidual é prejudicada, é observada mais comumente quando a gestação e o trabalho de parto são complicados por problemas que resultam em prejuízo no fornecimento de oxigênio para os tecidos fetais (Benesi, 1993; Vaala e House, 2006) e/ou devido à imaturidade pulmonar em nascimentos prematuros (Nowak, 1996). A asfixia periparto foi associada, entre outros fatores, à distocia, à indução do parto, à cesariana, à gemelaridade e à doença materna grave (Vaala, 1994), enquanto a asfixia no período neonatal pode resultar de disfunção cardiorrespiratória grave, de hipertensão pulmonar e da obstrução de vias aéreas.

Durante a asfixia, a redistribuição do fluxo sanguíneo para fora dos rins frequentemente ocasiona menor perfusão renal e necrose tubular aguda. Na medicina humana, a oligúria é o sinal clínico mais comum de insuficiência renal aguda, semelhante ao que também é observado em potros asfixiados (Koterba et al., 1990; Vaala, 1994). Outro indício de dano renal isquêmico pode ser obtido por meio do perfil bioquímico do animal, a partir de elevadas concentrações séricas de creatinina (Kaneko, 2008). O uso exógeno de corticoides durante o período prénatal em mulheres gestantes tem reduzido significativamente a incidência de bebês com "síndrome da angústia respiratória", o tempo de ventilação mecânica, o número de doses de surfactantes utilizados, além da ocorrência de quadros de hemorragia peri-intraventricular (Meneguel et al., 2003). Em cordeiros, os efeitos descritos são similares e podem ser justificados pelo aumento de lipídeos e proteínas do surfactante pulmonar (Ballard et al., 1997).

Anwar et al. (1999) também demonstraram outros efeitos associados ao uso de glicocorticoides em ovelhas gestantes, como aumento da pressão arterial média fetal, assim como aumento do hematócrito, da contagem de eritrócitos e da concentração de hemoglobina. Os autores verificaram que as contagens de neutrófilos e a concentração de proteínas totais dos fetos também foram maiores, contudo o número de linfócitos diminuiu significativamente em reposta à aplicação de betametasona.

Com este trabalho, objetivou-se comparar as variáveis hematológicas e o perfil bioquímico renal de cordeiros nascidos a termo e prematuros, ao longo das primeiras 48 horas de vida, bem como verificar a viabilidade desses prematuros mediante a administração materna de corticosteroide.

\section{MATERIAL E MÉTODOS}

Cordeiros oriundos de mães da raça Suffolk, em idade adulta, e provenientes de rebanho da Faculdade de Medicina Veterinária da Unesp Araçatuba, foram distribuídos em quatro grupos experimentais, a saber:

grupo PN: 15 cordeiros nascidos de partos normais (média de 146 dias de gestação);

grupo PNDEX: oito cordeiros nascidos de partos normais cujas mães receberam 16mg (Ingoldby e Jackson, 2001) de dexametasona (Azium ${ }^{\circledR}$, Schering-Plough), por via intramuscular, aos 141 dias de gestação (média de 143 dias);

grupo PRE: 10 cordeiros prematuros, nascidos por meio de cesarianas realizadas aos 138 dias de gestação;

grupo PREDEX: nove cordeiros prematuros, nascidos por meio de cesarianas realizadas aos 
138 dias de gestação, cujas mães receberam $16 \mathrm{mg}$ de dexametasona, por via intramuscular, dois dias antes da cirurgia (aos 136 dias).

As fêmeas pertencentes aos grupos PRE e PREDEX foram submetidas a cesarianas com o intuito de garantir o nascimento dos fetos no período preestabelecido de gestação (138 dias).

Na espécie ovina, o período gestacional varia de 145 a 148 dias (Jainudeen; Hafez, 2004). Os cordeiros nascidos a partir de 137 dias são considerados prematuros, porém quanto mais próxima a data do parto do período gestacional normal, em média 145 dias, maior a sobrevida desses animais (Mobini et al., 2004). Em estudos de modelo experimental para prematuros humanos, a interrupção da gestação ocorreu aos 133 dias nas ovelhas (Cock et al., 2005; De Matteo et al., 2009) e foi justificada pelos autores por ser a idade gestacional mínima para o nascimento de cordeiros viáveis sem a necessidade de suporte ventilatório. Entretanto, as informações são escassas com relação às espécies pecuárias. Radostits et al. (2002) citaram que essa idade mínima para a viabilidade dos recém-nascidos seria de 138 dias para a espécie ovina, período este que foi utilizado, no presente estudo, para o nascimento dos animais prematuros.

As datas de cobertura das ovelhas eram conhecidas e realizou-se exame ultrassonográfico (DP 2200 Vet, Mindray) abdominal para confirmação da gestação entre 45 e 60 dias após a última data de cobertura. $\mathrm{O}$ procedimento anestésico adotado nas cirurgias cesarianas foi realizado por meio de anestesia local com bloqueio paravertebral proximal nos ramos nervosos das vértebras T13, L1 e L2, utilizandose cloridrato de lidocaína (Xylestesin ${ }^{\circledR} 2 \%$, Cristália), no volume de $5 \mathrm{~mL}$ em cada ponto dorsal e ventral aos processos transversos. Adicionalmente, associou-se a anestesia peridural lombossacra (L6- S1) com sulfato de morfina $\left(\right.$ Dimorf $^{\circledR}$, Cristália) na dose de $0,1 \mathrm{mg} / \mathrm{kg}$ diluída em $5 \mathrm{~mL}$ de solução fisiológica. Nos casos em que a anestesia paravertebral não foi eficiente, realizou-se bloqueio infiltrativo no local da incisão com cloridrato de lidocaína. Após a retirada do feto, quando necessário, as ovelhas recebiam sedação com maleato de midazolam (Dormonid ${ }^{\circledR}$, Roche) na dose de $0,2 \mathrm{mg} / \mathrm{kg}$. O procedimento cirúrgico foi realizado com as ovelhas colocadas em decúbito lateral direito, para incisão em região do flanco esquerdo, conforme técnica descrita por Tibary e Van Metre (2004).

Os cordeiros provenientes de partos normais permaneceram com as mães, ingerindo colostro à vontade. Os animais oriundos de cesarianas foram acompanhados e alimentados com colostro proveniente de banco de colostro bovino, devido à ausência de produção de colostro nas mães e à dificuldade para formação de banco de colostro ovino. O colostro foi fornecido por intermédio do uso de mamadeiras nas primeiras horas de vida; quando não apresentavam reflexo de sucção, a administração era realizada com auxílio de sonda nasoesofágica.

Os cuidados referentes ao suporte ventilatório, quando do desenvolvimento de hipóxia, foram realizados mantendo-se os prematuros sob ventilação assistida durante período máximo padronizado de 30 minutos.

Amostras de sangue venoso foram colhidas logo após o nascimento (M0h), aos 15 minutos (M15min), aos 60 minutos (M60min), às 24 horas (M24h) e às 48 horas de vida (M48h). Após antissepsia local, realizou-se punção da veia jugular, utilizando-se agulhas $25 \times 0,7 \mathrm{~mm}$ acopladas a tubos Vacutainer $^{\circledR}$ com anticoagulante ácido etilenodiamino tetracético (EDTA), para volume de $5 \mathrm{~mL}$, e tubos Vacutainer $^{\circledR}$ siliconizados sem anticoagulante, para volume de $10 \mathrm{~mL}$. O sangue recolhido para obtenção do soro foi mantido em temperatura ambiente, ao abrigo da luz, até a coagulação e retração do coágulo. Em seguida, realizou-se centrifugação a $1.000 \mathrm{G}$, durante cinco minutos, para melhor separação do soro. Este foi transferido para frascos de plástico apropriados, divididos em três alíquotas, com auxílio de pipeta automática, e congelado imediatamente a $20^{\circ} \mathrm{C}$, até o momento do seu processamento.

O teor de hemoglobina foi mensurado em espectrofotômetro semiautomático (Labquest, Labtest, Belo Horizonte, Minas Gerais), empregando-se o reativo comercial de cianometa-hemoglobina. O volume globular foi obtido com a utilização de tubos capilares e centrífuga para micro-hematócrito, sendo as amostras de sangue com EDTA centrifugadas a 13.000G, durante cinco minutos. 
A concentração plasmática de proteína total foi determinada utilizando-se refratômetro clínico (Master-SUR/NM, ATAGO, Tóquio, Japão), e o teor plasmático de fibrinogênio obtido pelo método de precipitação pelo calor e pela leitura em refratômetro (Millar et al., 1971).

O leucograma foi realizado mediante a contagem leucocitária manual, em câmara de Neubauer (Neubauer Improved Bright-Lined, New Optik), diluindo-se a amostra com a utilização de pipetas apropriadas (Pipeta de Thoma, Writeg, Alemanha). Para cada amostra colhida, preparouse esfregaço sanguíneo corado, para a contagem de 100 células, com corante panótico rápido (LaborClin) para a contagem diferencial de leucócitos (Garcia-navarro, 1994).

As concentrações séricas de ureia e creatinina foram mensuradas em espectrofotômetro semiautomático (Labquest, Labtest, Belo Horizonte, MG), utilizando-se conjuntos de reagentes de uso comercial (Labtest, Belo Horizonte, MG).

Os dados foram submetidos à análise de variância com medidas repetidas, sendo as médias comparadas pelo teste de Tukey no nível de significância de $5 \%$. As variáveis concentração plasmática de fibrinogênio e contagens total e diferencial de leucócitos foram analisadas pelo teste de Kruskal-Wallis para comparar os grupos em cada momento e pelo teste de Friedman para comparar os momentos em cada grupo, seguido do teste de Dunn para comparações múltiplas. As análises estatísticas foram efetuadas empregando-se o programa SAS (Statistical..., 2008), sendo consideradas significativas quando $\mathrm{P}<0,05$ (Zar, 1998).

O presente estudo foi submetido à avaliação pelo Comitê de Ética Animal da Faculdade de Medicina Veterinária, Unesp/Araçatuba (Committee for Ethical Use of Animals - Ceua, protocolo 02493-2011).

\section{RESULTADOS E DISCUSSÃO}

Nos grupos PN e PNDEX foram avaliados 15 e oito cordeiros, respectivamente, do nascimento às 48 horas de vida. Os grupos PRE e PREDEX começaram com 10 e nove cordeiros. Contudo, em virtude da ocorrência de vários óbitos com a evolução do tempo, restaram, finalizado o período de observação do estudo, apenas três e sete cordeiros, nos respectivos grupos $(70 \% \mathrm{e}$ $22,22 \%$ de mortalidade nos grupos PRE e PREDEX). Estes animais foram encaminhados ao setor de Patologia Veterinária da FMVA/Unesp e, à necropsia, foram identificados pulmões congestos com áreas de atelectasia em graus variados, com causa mortis identificada como insuficiência cardiorrespiratória.

De acordo com Dawes e Parry (1965), a taxa de mortalidade de cordeiros nascidos de forma prematura sem qualquer indução médica é consideravelmente reduzida, em torno de $19 \%$. Em contraste, o índice de sobrevivência de cordeiros provenientes de ovelhas que tiveram seus partos induzidos com dexametasona tende a aumentar drasticamente, chegando a $81 \%$ (Zoller et al., 2015). Essa discrepância pode ser justificada pelos efeitos dos corticosteroides no aparecimento acelerado do surfactante, exercendo influência direta na expansão parcial dos pulmões (Liggins, 1969). Nos cordeiros com menos de 120 dias de idade gestacional, o padrão de deflação pulmonar é caracterizado por pequeno aprisionamento de ar e colapso completo, acompanhado de baixa pressão (Brumley et al., 1967).

É importante ressaltar que resultados positivos após a administração materna de glicocorticoides dependem da fase gestacional e do desenvolvimento pulmonar (Jobe, 2001; Bonanno; Wapner, 2012), o que é corroborado pela ocorrência de $22,2 \%$ de mortalidade no grupo PREDEX.

Os resultados deste estudo mostram que a mortalidade é elevada $(70 \%)$ e a vitalidade nos sobreviventes é menor em cordeiros nascidos uma semana antes (aos 138 dias) do período gestacional médio, aproximadamente, para a referida espécie (145 dias de gestação, Mobini et al., 2004). Embora haja informação na literatura sobre a idade gestacional mínima que garantiria a sobrevivência das diferentes espécies, sendo, geralmente, de 138 dias para ovinos (Radostits et al., 2002), o presente trabalho contesta tal informação, em virtude da elevada taxa de mortalidade verificada logo após o nascimento ou durante os primeiros 15 minutos de vida. A interrupção da gestação aos 138 dias provavelmente comprometeu a produção de surfactante, uma vez que foram observadas áreas 
de atelectasia pulmonar, o que indica que não houve expansão dos alvéolos ou esta não ocorreu de maneira eficiente (Cuningham, 2004).

Os valores médios do volume globular (VG) e do teor de hemoglobina $(\mathrm{Hb})$ diminuíram ao longo do período de observação, nos quatro grupos experimentais (Fig. 1 e 2). No M0h e M15min, os cordeiros do grupo PREDEX apresentaram valores mais baixos de VG em relação ao grupo $\mathrm{PN}$, enquanto para a variável $\mathrm{Hb}$ constatou-se diferença entre grupos apenas no M15, sendo os valores médios de PREDEX também inferiores aos do grupo PN. Entretanto, os resultados estiveram dentro dos limites considerados fisiológicos para animais adultos da espécie ovina (Jain, 1993).

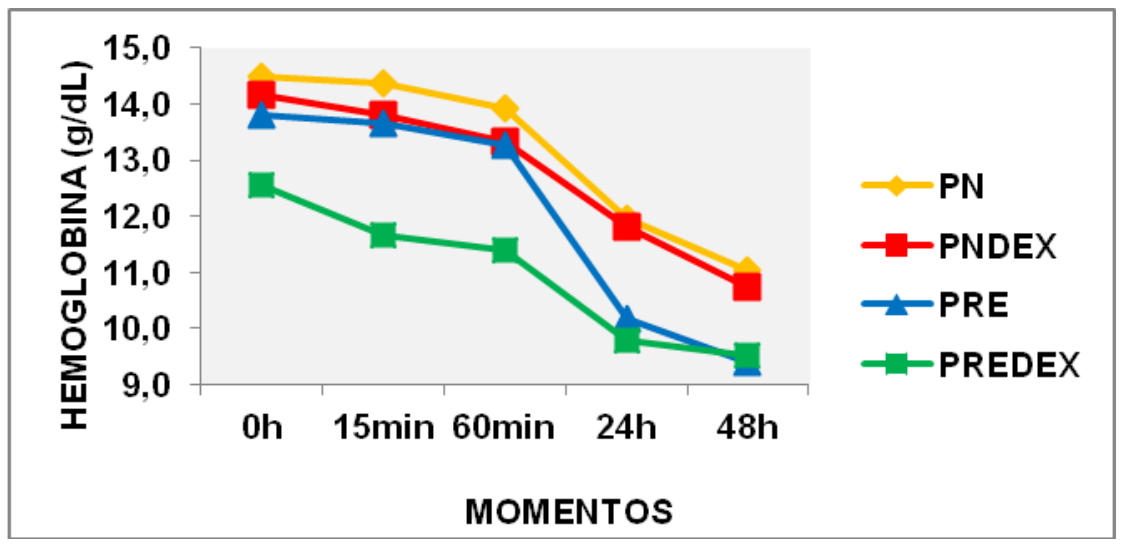

Figura 1. Representação gráfica dos valores médios do teor de hemoglobina ( $\mathrm{g} / \mathrm{dL})$, desde o nascimento até as 48 horas de vida, em cordeiros nascidos de parto normal (PN), nascidos de parto normal cujas mães receberam dexametasona aos 141 dias de gestação (PNDEX), nascidos prematuramente por cesariana aos 138 dias de gestação (PRE) e nascidos prematuramente aos 138 dias de gestação cujas mães receberam dexametasona dois dias antes (PREDEX).

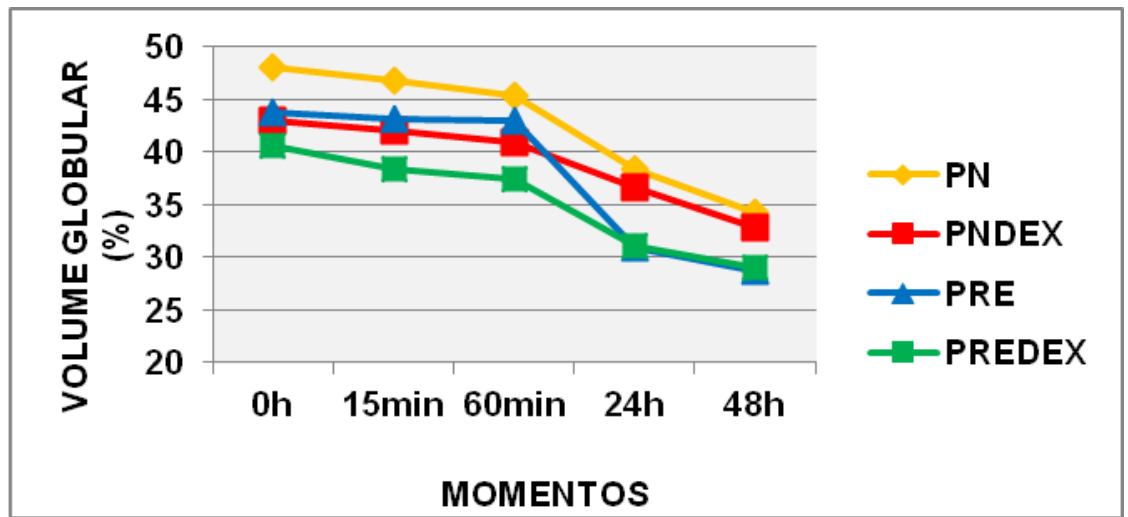

Figura 2. Representação gráfica dos valores médios do volume globular (\%), desde o nascimento até as 48 horas de vida, em cordeiros nascidos de parto normal (PN), nascidos de parto normal cujas mães receberam dexametasona aos 141 dias de gestação (PNDEX), nascidos prematuramente por cesariana aos 138 dias de gestação (PRE) e nascidos prematuramente aos 138 dias de gestação cujas mães receberam dexametasona dois dias antes (PREDEX).

Probo et al. (2012) encontraram valores mais altos de VG e $\mathrm{Hb}$ em bezerros nascidos por cesariana e correlacionaram-nos a mudanças no padrão hematológico decorrentes de diferentes níveis de oxigenação sanguínea. Tal fato parece ficar mais evidente na síndrome do desconforto respiratório, a qual, segundo os autores, pode estar associada à realização de cirurgias 
cesarianas. É importante ressaltar, todavia, que, no presente estudo, as ovelhas estavam em gestação normal, sem a necessidade de indicação de cesariana, tendo esta sido realizada somente para promover o nascimento dos cordeiros de maneira prematura. Assim, a condição de hipóxia e hipercapnia no ambiente uterino, considerada fisiológica para todos os animais, provavelmente não tenha sido suficiente para exacerbar qualquer efeito sobre os parâmetros hematológicos citados. Embora outros autores tenham mencionado a interferência do tipo de parto sobre o equilíbrio ácido-básico de animais recém-nascidos (Bovino, 2011; Camargo, 2010; Szenci et al., 1989), as variáveis VG e Hb não apresentaram alterações tão expressivas.

Os maiores valores de VG e Hb logo após o nascimento podem ser explicados, segundo Benesi (1993), pela incorporação de sangue placentário ao dos animais recém-nascidos, além do estresse causado pelo nascimento, que se reflete com a elevação das concentrações plasmáticas de glicocorticoides, elevando a pressão sanguínea com maior mobilização de eritrócitos das reservas orgânicas, o que aumenta seu número circulante. $O$ autor cita que a variação subsequente nos parâmetros do eritrograma deve-se à intensa mobilização dos eritrócitos fetais e à hemodiluição por modificação dos fluidos corporais após o nascimento; tal fato pode explicar o mesmo perfil de diminuição dos valores médios das variáveis hematológicas neste estudo ao longo das 48 horas.

A concentração plasmática de proteínas totais (PT) mostrou variação significativa em todos os momentos avaliados (Fig. 3), com os menores valores médios encontrados no grupo PRE. Nos quatro grupos experimentais, verificou-se aumento da concentração plasmática de PT no M24h e M48h, coincidindo com os momentos de maior ingestão láctea. Os animais pecuários nascem praticamente desprovidos de imunidade e necessitam da ingestão frequente de carboidratos prontamente utilizáveis para manter a energia corpórea (Stull e Reynolds, 2008). O aumento na concentração plasmática de PT no M24h evidencia a influência positiva do colostro sobre os cordeiros. Porém, percebeu-se que os animais prematuros (PRE e PREDEX) tiveram menores valores médios de PT, o que denota provável dificuldade na capacidade de absorção das imunoglobulinas maternas.

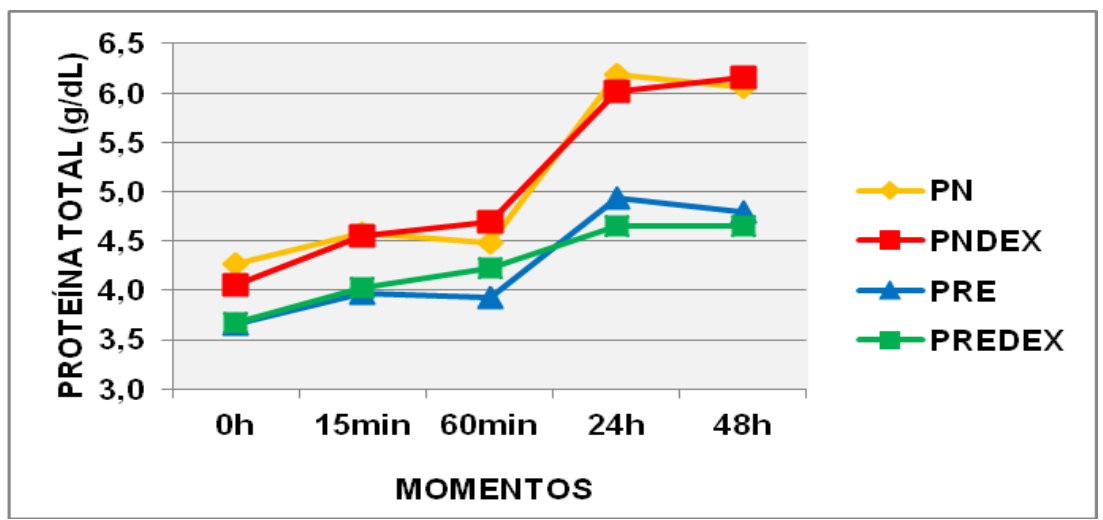

Figura 3. Representação gráfica dos valores médios da concentração plasmática de proteínas totais (g/dL) desde o nascimento até as 48 horas de vida, em cordeiros nascidos de parto normal (PN), nascidos de parto normal cujas mães receberam dexametasona aos 141 dias de gestação (PNDEX), nascidos prematuramente por cesariana aos 138 dias de gestação (PRE) e nascidos prematuramente aos 138 dias de gestação cujas mães receberam dexametasona dois dias antes (PREDEX).

Estudos em recém-nascidos humanos demonstraram sua capacidade precoce em esquematizar resposta inflamatória eficiente ainda durante o trabalho de parto (Steinborn et al., 1999; Marchini et al., 2000). Nesse contexto, seria possível imaginar que houvesse aumento nas concentrações plasmáticas de proteínas inflamatórias. Entretanto, no presente estudo, as concentrações plasmáticas de fibrinogênio, uma importante proteína positiva de fase aguda, apresentaram o mesmo perfil em todos os grupos, sem variações expressivas nos diferentes 
momentos (Tab. 4). A ventilação mecânica em cordeiros prematuros, utilizados como modelo experimental para humanos, foi associada à ocorrência de lesões pulmonares alveolares (Hillman et al., 2010; Hillman et al., 2011), o que poderia justificar o aumento da concentração de proteínas inflamatórias. Ressalta-se, entretanto, que, no presente estudo, $70 \%$ dos animais do grupo PRE vieram a óbito logo após o nascimento ou nos primeiros 15 minutos de vida, mesmo sendo submetidos às medidas de reanimação neonatal; portanto, dificilmente lesões decorrentes do processo de ventilação, realizado no presente estudo, seriam responsáveis por alterações expressivas nas proteínas de fase aguda.

Tabela 4. Mediana (Md), mínimo (Mín) e máximo (Máx) da concentração plasmática de fibrinogênio $(\mathrm{g} / \mathrm{dL})$ em cordeiros nascidos de parto normal (PN), nascidos de parto normal cujas mães receberam dexametasona aos 141 dias de gestação (PNDEX), nascidos prematuramente por cesariana aos 138 dias de gestação (PRE) e nascidos prematuramente aos 138 dias de gestação cujas mães receberam dexametasona 48 horas antes (PREDEX) desde o nascimento até as 48 horas de vida

\begin{tabular}{|c|c|c|c|c|c|c|c|c|c|c|c|c|}
\hline \multirow{3}{*}{ Momento } & & & \multicolumn{10}{|c|}{ Fibrinogênio (g/dL) } \\
\hline & \multicolumn{3}{|c|}{$\mathrm{PN}$} & \multicolumn{3}{|c|}{ PNDEX } & \multicolumn{3}{|c|}{ PRE } & \multicolumn{3}{|c|}{ PREDEX } \\
\hline & $\mathrm{N}$ & Md & Mín-Máx & $\mathrm{N}$ & Md & Mín-Máx & $\mathrm{N}$ & Md & Mín-Máx & $\mathrm{n}$ & Md & Mín-Máx \\
\hline $\mathrm{M}_{0 \mathrm{~h}}$ & 15 & $<0,1$ & $<0,1-0,4$ & 8 & $<0,1$ & $<0,1-0,2$ & 10 & 0,1 & $<0,1-0,4$ & 9 & $<0,1$ & $<0,1-0,2$ \\
\hline $\mathbf{M}_{15 \min }$ & 15 & $<0,1$ & $<0,1-0,2$ & 8 & $<0,1$ & $<0,1-0,2$ & 7 & $<0,1$ & $<0,1-0,4$ & 8 & $<0,1$ & $<0,1-0,2$ \\
\hline $\mathrm{M}_{60 \mathrm{~min}}$ & 15 & $<0,1$ & $<0,1-0,2$ & 8 & $<0,1$ & $<0,1-0,2$ & 5 & $<0,1$ & $<0,1-0,2$ & 8 & $<0,1$ & $<0,1-0,2$ \\
\hline $\mathrm{M}_{24 \mathrm{~h}}$ & 15 & 0,2 & $<0,1-0,6$ & 8 & 0,1 & $<0,1-0,2$ & 3 & $<0,1$ & $<0,1-0,2$ & 8 & 0,2 & $0,2-0,4$ \\
\hline $\mathrm{M}_{48 \mathrm{~h}}$ & 15 & 0,2 & $<0,1-0,8$ & 8 & 0,1 & $<0,1-0,4$ & 3 & 0,2 & $0,2-0,4$ & 7 & 0,2 & $<0,1-0,4$ \\
\hline
\end{tabular}

Grönlund et al. (1999) sugeriram que o tipo de parto seria capaz de modificar o equilíbrio imunológico no recém-nascido. Nesse contexto, bebês nascidos de cesarianas após longo trabalho de parto apresentaram contagens mais altas de leucócitos e neutrófilos quando comparados às cesarianas eletivas, dados corroborados por Redüko et al. (2005) e Probo et al. (2012). Os resultados aqui apresentados, porém, discordam de tais achados, uma vez que a contagem leucocitária foi mais alta no grupo $\mathrm{PN}$ em relação aos demais (Tab. 5), porém de maneira significativa apenas no M24h. Ao longo do período de observação, apenas o grupo $\mathrm{PN}$ mostrou diferença entre o M24h e os demais momentos, semelhantemente ao que Benesi (1993) verificou em bezerros sadios, cuja contagem total de leucócitos tendeu a diminuir a partir das 24 horas de vida. Com relação à contagem diferencial de leucócitos, verificou-se que o grupo PRE apresentou os menores valores de neutrófilos em relação aos demais no M0h, M15min e M60min. Não houve diferenças nas contagens de linfócitos e monócitos nos diferentes grupos e momentos.

Tabela 5. Mediana (Md), mínimo (Mín) e máximo (Máx) das contagens total e diferencial de leucócitos $\left(\mathrm{x} 10^{3} / \mu \mathrm{L}\right)$ em cordeiros nascidos de parto normal $(\mathrm{PN})$, nascidos de parto normal cujas mães receberam dexametasona aos 141 dias de gestação (PNDEX), nascidos prematuramente por cesariana aos 138 dias de gestação (PRE) e nascidos prematuramente aos 138 dias de gestação cujas mães receberam dexametasona 48 horas antes (PREDEX) desde o nascimento até as 48 horas de vida

\begin{tabular}{|c|c|c|c|c|c|c|c|c|c|c|c|c|c|}
\hline \multirow[b]{2}{*}{ Variável } & \multirow[b]{2}{*}{ Momento } & \multicolumn{3}{|c|}{ PN } & \multicolumn{3}{|c|}{ PNDEX } & \multicolumn{3}{|c|}{ PRE } & \multicolumn{3}{|c|}{ PREDEX } \\
\hline & & $\mathrm{N}$ & Md & $\begin{array}{l}\text { Mín- } \\
\text { Max }\end{array}$ & $\mathrm{N}$ & $\mathrm{Md}$ & $\begin{array}{l}\text { Mín- } \\
\text { Max }\end{array}$ & $\mathrm{N}$ & Md & $\begin{array}{l}\text { Mín- } \\
\text { Max }\end{array}$ & $\mathrm{n}$ & Md & Mín-Max \\
\hline \multirow{5}{*}{$\begin{array}{l}\text { Leucócitos } \\
\left(\times 10^{3} / \mu \mathrm{L}\right)\end{array}$} & $\mathrm{M}_{0 \mathrm{~h}}$ & 15 & $2,7 b$ & $0,8-7,6$ & 8 & 3,3 & $0,7-4,2$ & 10 & 2,2 & $1,1-4,5$ & 7 & 2,2 & $1,4-3,7$ \\
\hline & $\mathbf{M}_{15 \min }$ & 15 & $2,4 b$ & $1,1-7,0$ & 8 & 3,6 & $0,7-4,4$ & 7 & 2,2 & $0,9-3,6$ & 9 & 3,4 & $1,5-5,1$ \\
\hline & $\mathbf{M}_{60 \min }$ & 15 & $2,1 b$ & $1,2-4,5$ & 8 & 2,8 & $1,2-4,2$ & 5 & 2,0 & $1,5-2,9$ & 8 & 2,7 & $1,05,5$ \\
\hline & $\mathrm{M}_{24 \mathrm{~h}}$ & 15 & $3,8 \mathrm{Aa}$ & $2,7-6,1$ & 8 & $2,7 \mathrm{~B}$ & $1,5-3,5$ & 3 & $2,2 \mathrm{~B}$ & $1,7-3,2$ & 8 & $2,3 \mathrm{~B}$ & $0,8-3,7$ \\
\hline & $\mathrm{M}_{48 \mathrm{~h}}$ & 15 & $2,9 \mathrm{ab}$ & $1,1-6,4$ & 8 & 2,9 & $1,5-3,0$ & 3 & 2,5 & $1,7-2,8$ & 8 & 2,0 & $1,6-3,0$ \\
\hline \multirow{5}{*}{$\begin{array}{c}\text { Neutrófilos } \\
\left(\times 10^{3} / \mu \mathrm{L}\right)\end{array}$} & $\mathrm{M}_{0 \mathrm{~h}}$ & 15 & $1,1 \mathrm{~A}$ & $0,3-4,7$ & 8 & $1,5^{\mathrm{a}}$ & $0,2-2,4$ & 10 & $0,2 \mathrm{~B}$ & $0,04-0,9$ & 9 & $0,7 \mathrm{AB}$ & $0,2-1,6$ \\
\hline & $\mathrm{M}_{15 \min }$ & 15 & $0,9 \mathrm{~A}$ & $0,4-3,9$ & 8 & $1,0 \mathrm{~A}$ & $0,1-2,3$ & 7 & $0,1 \mathrm{~B}$ & $0,02-1,1$ & 8 & $0,7 \mathrm{AB}$ & $0,02-1,7$ \\
\hline & $\mathrm{M}_{60 \mathrm{~min}}$ & 15 & $0,9 \mathrm{~A}$ & $0,4-2,7$ & 8 & $1,0 \mathrm{~A}$ & $0,3-2,6$ & 5 & $0,3 \mathrm{~B}$ & $0,1-0,8$ & 8 & $0,4 \mathrm{AB}$ & $0,2-3,6$ \\
\hline & $\mathrm{M}_{24 \mathrm{~h}}$ & 15 & 2,4 & $0,1-4,9$ & 8 & 1,3 & $0,5-2,4$ & 3 & 1,5 & $0,8-2,1$ & 8 & 1,2 & $0,4-2,9$ \\
\hline & $\mathrm{M}_{48 \mathrm{~h}}$ & 15 & 1,2 & $0,2-4,4$ & 8 & 1,2 & $0,3-2,0$ & 3 & 1,5 & $0,9-1,5$ & 7 & 0,5 & $0,2-1,1$ \\
\hline
\end{tabular}

Medianas seguidas de letras diferentes, maiúscula na linha e minúscula na coluna, diferem entre si $(\mathrm{P}<0,05)$. A ausência de letras implica que não há diferença estatística. 
O perfil bioquímico renal mostrou variações nas concentrações séricas de ureia e creatinina (Tab. 6 e 7), denotando a imaturidade dos sistemas orgânicos logo após o nascimento. Os valores médios da concentração sérica de ureia oscilaram dentro da faixa de normalidade para ovinos adultos, mas também variaram acima do limite superior (Jain, 1993). As concentrações séricas de creatinina foram significativamente mais altas no grupo PRE em relação ao PNDEX no M60min, M24h e M48h e, em relação ao PN, no M24h e M48h. Durante a asfixia, a redistribuição do fluxo sanguíneo frequentemente ocasiona menor perfusão renal e necrose tubular aguda
(Koterba et al., 1990; Vaala, 1994). Em todos os grupos, as concentrações séricas de creatinina diminuíram no M24h e M48h, semelhantemente ao observado por Feitosa et al. (2009), provavelmente em consequência do contínuo processo de maturação funcional que ocorre após o nascimento, tendendo à normalização dos parâmetros bioquímicos. Assim, ainda que tenha havido comprometimento renal, mediante a hipóxia que acometeu os animais prematuros nos primeiros minutos de vida, esse processo provavelmente se desenvolveu em graus variados, sendo mais leve nos recém-nascidos sobreviventes.

Tabela 6. Média ( $\overline{\mathrm{X}}$ ) e desvio-padrão (SD) da concentração sérica de ureia (mg/dL) em cordeiros nascidos de parto normal (PN), nascidos de parto normal cujas mães receberam dexametasona aos 141 dias de gestação (PNDEX), nascidos prematuramente por cesariana aos 138 dias de gestação (PRE) e nascidos prematuramente aos 138 dias de gestação cujas mães receberam dexametasona 48 horas antes (PREDEX) desde o nascimento até as 48 horas de vida

\begin{tabular}{|c|c|c|c|c|c|c|c|c|}
\hline \multirow{3}{*}{ Momento } & \multicolumn{8}{|c|}{ Ureia $(\mathrm{mg} / \mathrm{dL})$} \\
\hline & \multicolumn{2}{|r|}{$\mathrm{PN}$} & \multicolumn{2}{|r|}{ PNDEX } & \multicolumn{2}{|r|}{ PRE } & \multicolumn{2}{|r|}{ PREDEX } \\
\hline & $\mathrm{N}$ & $\bar{x}_{ \pm S}$ & $\mathrm{n}$ & $\bar{X}_{ \pm S}$ & $\mathrm{n}$ & $\bar{X}_{ \pm S}$ & $\mathrm{n}$ & $\bar{X}_{ \pm S}$ \\
\hline $\mathrm{M}_{0 \mathrm{~h}}$ & 15 & $39,71 \pm 11,2 \mathrm{Bb}$ & 8 & $57,15 \pm 13,6 \mathrm{~A}$ & 10 & $\begin{array}{l}46,30 \pm 15,9 \\
\mathrm{ABab}\end{array}$ & 8 & $\begin{array}{l}62,37 \pm 17,0 \\
\mathrm{Aa}\end{array}$ \\
\hline $\mathbf{M}_{15 \min }$ & 15 & $42,60 \pm 11,2 b$ & 8 & $55,34 \pm 12,8$ & 7 & $46,99 \pm 14,8 \mathrm{ab}$ & 8 & $58,43 \pm 14,6 \mathrm{a}$ \\
\hline $\mathrm{M}_{60 \mathrm{~min}}$ & 15 & $43,77 \pm 11,3 b$ & 8 & $58,09 \pm 12,8$ & 5 & $42,54 \pm 8,6 b$ & 8 & $59,73 \pm 16,6 a$ \\
\hline $\mathrm{M}_{24 \mathrm{~h}}$ & 15 & $75,69 \pm 26,7 \mathrm{a}$ & 8 & $55,77 \pm 19,1$ & 3 & $63,24 \pm 2,4 \mathrm{a}$ & 8 & $58,29 \pm 18,9$ a \\
\hline $\mathrm{M}_{48 \mathrm{~h}}$ & 15 & $68,24 \pm 31,9 \mathrm{Aa}$ & 8 & $56,08 \pm 18,9 \mathrm{AB}$ & 3 & $\begin{array}{l}54,27 \pm 20,3 \\
\mathrm{ABab}\end{array}$ & 7 & $\begin{array}{l}35,84 \pm 10,2 \\
\mathrm{Bb}\end{array}$ \\
\hline
\end{tabular}

Médias seguidas de letras diferentes, maiúscula na linha e minúscula na coluna, diferem entre si pelo teste de Tukey $(\mathrm{P}<0,05)$. A ausência de letras implica que não há diferença estatística.

Tabela 7. Média ( $\overline{\mathrm{X}}$ ) e desvio-padrão (SD) da concentração sérica de creatinina (mg/dL) de cordeiros nascidos de parto normal (PN), nascidos de parto normal cujas mães receberam dexametasona aos 141 dias de gestação (PNDEX), nascidos prematuramente por cesariana aos 138 dias de gestação (PRE) e nascidos prematuramente aos 138 dias de gestação cujas mães receberam dexametasona 48 horas antes (PREDEX) desde o nascimento até as 48 horas de vida.

\begin{tabular}{|c|c|c|c|c|c|c|c|c|}
\hline \multirow{3}{*}{ Momento } & \multicolumn{8}{|c|}{ Creatinina (mg/dL) } \\
\hline & \multicolumn{2}{|r|}{$\mathrm{PN}$} & \multicolumn{2}{|r|}{ PNDEX } & \multicolumn{2}{|c|}{ PRE } & \multicolumn{2}{|r|}{ PREDEX } \\
\hline & $\mathrm{n}$ & $\bar{x}_{ \pm S}$ & $\mathrm{~N}$ & $\bar{x}_{ \pm S}$ & $\mathrm{~N}$ & $\overline{\mathrm{X}}_{ \pm S}$ & $\mathrm{n}$ & $\bar{x}_{ \pm S}$ \\
\hline $\mathrm{M}_{0 \mathrm{~h}}$ & 15 & $2,20 \pm 0,9 \mathrm{a}$ & 8 & $1,66 \pm 0,7 \mathrm{a}$ & 10 & $2,40 \pm 0,5 \mathrm{a}$ & 8 & $2,00 \pm 0,2 \mathrm{a}$ \\
\hline $\mathrm{M}_{15 \min }$ & 15 & $1,94 \pm 0,9 \mathrm{a}$ & 8 & $1,67 \pm 0,6 \mathrm{a}$ & 7 & $2,56 \pm 0,6 \mathrm{a}$ & 8 & $1,97 \pm 0,2 \mathrm{a}$ \\
\hline $\mathrm{M}_{60 \mathrm{~min}}$ & 15 & $1,85 \pm 0,8 \mathrm{Aba}$ & 8 & $1,58 \pm 0,7 \mathrm{Ba}$ & 5 & $2,60 \pm 0,7 \mathrm{Aa}$ & 8 & $1,90 \pm 0,3 \mathrm{Aba}$ \\
\hline $\mathrm{M}_{24 h}$ & 15 & $0,80 \pm 0,3 \mathrm{Bb}$ & 8 & $0,74 \pm 0,1 \mathrm{Bb}$ & 3 & $1,13 \pm 0,2 \mathrm{Ab}$ & 8 & $0,81 \pm 0,1 \mathrm{ABb}$ \\
\hline $\mathrm{M}_{48 \mathrm{~h}}$ & 15 & $0,65 \pm 0,2 \mathrm{Bb}$ & 8 & $0,85 \pm 0,3 \mathrm{Bb}$ & 3 & $1,12 \pm 0,6 \mathrm{Ab}$ & 7 & $0,73 \pm 0,1 \mathrm{ABb}$ \\
\hline
\end{tabular}

Médias seguidas de letras diferentes, maiúscula na linha e minúscula na coluna, diferem entre si pelo teste de Tukey $(\mathrm{P}<0,05)$. A ausência de letras implica que não há diferença estatística. 


\section{CONCLUSÕES}

Os parâmetros avaliados foram afetados pela prematuridade na espécie ovina, e a dexametasona, embora nem sempre efetiva sobre as variáveis estudadas, teve influência positiva sobre a taxa de sobrevivência dos animais prematuros.

\section{AGRADECIMENTOS}

Os autores agradecem o aporte financeiro da Fundação de Amparo à Pesquisa do Estado de São Paulo (Fapesp) para o desenvolvimento do projeto (Processo 2010/19497-4) e a concessão de bolsas de doutorado (Processo 2011/01123-3).

\section{REFERÊNCIAS}

ANWAR, M.A.; SCHWAB, M.; POSTON, L.; NATHANIELSZ, P. W. Betamethasone-mediated vascular dysfunction and changes in hematological profile in the ovine fetus. Am. J. Physiol. Heart Circ. Physiol., v.276, p.1137-1143, 1999. BALLARD, P.L.; NING, Y.; POLK, D. et al. Glucocorticoid regulation of surfactant components in immature lambs. Am. $J$. Physiol. Lung Cell. Mol. Physiol., v.273, p.1048-1057, 1997.

BENESI, F.J. Hematologia de bezerros recémnascidos. Influência da asfixia neonatal, do tipo do parto e da ingestão de colostro sobre a crase sanguínea. 1993. 126f. Tese (Livre-Docência) Faculdade de Medicina Veterinária e Zootecnia, Universidade de São Paulo, São Paulo, SP.

BONANNO, C.; WAPNER, R.J. Antenatal corticosteroids in the management of preterm birth: are we back where we started? Obstet. Gynecol. Clin. N. Am., v.39, p.47-63, 2012.

BOVINO, F. Determinação do escore Apgar, dos valores hemogasométricos e do proteinograma sérico em cordeiros (Ovis aries) nascidos de partos normais e de cesarianas. 2011. 86f. Dissertação (Mestrado em Medicina veterinária) - Faculdade de Medicina Veterinária, Universidade Estadual Paulista, Araçatuba, SP.

BRUMLEY, G.W.; CHERNICK, V.; HODSON, W.A. et al. Correlations of mechanical stability, morphology, pulmonary surfactant, and phospholipid content in the developing lamb lung. J. Clin. Invest., v.46, p.863-873, 1967.
CAMARGO, D. G. Avaliação do sistema Apgar (modificado por Born, 1981) e dos níveis de cortisolemia, glicemia e de gases sanguíneos em cabritos nascidos de partos eutócicos e de cesariana. 2010. 93f. Dissertação (Mestrado) - Faculdade de Medicina Veterinária, Universidade Estadual Paulista, Araçatuba, SP.

CAMBIER, C.; CLERBAUX, T.; DETRY, B. et al. Blood oxygen binding in double-muscled calves and dairy calves with conventional conformation. Am. J. Vet. Res., v.61, p.299-304, 2000.

COCK, M.; HANNA, M.; SOZO, F. et al. Pulmonary function and structure following mild preterm birth in lambs. Pediatr. Pulmonol., v.40, p.336-348, 2005.

CUNNINGHAM, J.G. Transporte de oxigênio fetal e neonatal. In: _. Tratado de Fisiologia veterinária. Rio de Janeiro: Guanabara Koogan, 2004. p.533-538.

DAWES, G.S.; PARRY,H.B. Premature delivery and survival in lambs. Nature, v.207, p.330, 1965.

DE MATTEO, R.; SNIBSON, K.; THOMPSON, B. et al. Lung function in developing lambs: is it affected by preterm birth? J. Appl. Physiol., v.107, p.1083$1088,2009$.

FEITOSA, F.L.F.; PEIRÓ, J.R.; MENDES, L.C.N. et al. Determinação do perfil bioquímico renal sérico de bezerros holandeses e mestiços, na região de Araçatuba/SP In: CONGRESSO BRASILEIRO DE BUIATRIA, 8., 2009, Belo Horizonte. Anais... Belo Horizonte: [ABB], 2009. p.255-259. (suplemento).

GARCIA-NAVARRO, C.E.K. (Ed.). Manual de hematologia veterinária. São Paulo: VARELA, 1994, $169 \mathrm{p}$.

GASPARONI, A.; MACCARIO, R.; CHIRICO, G. et al. Neonatal B lymphocyte subpopulation and method of delivery. Biol. J. Neonatol., v.61, p.137-141, 1992.

GRÖNLUND, M.M.; NUUTILA, J.; PELTO, L. et al. Mode of delivery directs the phagocyte functions of infants for the first 6 months of life. Clin. Exp. Immunol., v.116, p.521-526, 1999.

HERSON, V.C.; BLOCK, C.; EISENFELD, L.I. et al. Effect of labor and delivery on neonatal polymorphonuclear leukocyte number and function. Am. J. Perinatol., v.9, p.285-288, 1992.

HILLMAN, N.H.; KALLAPUR, S.G.; PILOW, J.J. et al. Airway injury from initiating ventilation in preterm sheep. Pediatr. Res., v.67, p.60-65, 2010.

HILLMAN, N.H.; POLGLASE, G.R.; PILLOW, J.J. et al. Inflammation and lung maturation from stretch injury in preterm fetal sheep. Am. J. Physiol. Lung Cell. Mol. Physiol., v.300, p.232-241, 2011. 
INGOLDBY, L.; JACKSON, P. Induction of parturition in sheep. In Practice, v.23, p.228-231, 2001 .

JAIN, N.C. Essentials of veterinary hematology. Pennsylvania: Lea \& Febiger, 1993. 417 p.

JAINUDEEN, M.R.; HAFEZ, E.S.E. Gestação, fisiologia pré-natal e parto. In: HAFEZ, B.; HAFEZ, E.S.E. Reprodução animal. 7.ed. Barueri: Manole, 2004, p.141-155.

JAINUDEEN, M.R.; HAFEZ, E.S.E. Gestação, fisiologia pré-natal e parto. In: HAFEZ, B.; HAFEZ, E.S.E. Reprodução animal. 7.ed. Barueri: Manole, 2004, p.141-155.

JOBE, A.H. Glucocorticoids, inflammation and the perinatal lung. Semin. Neonatol., v.6, p.331-342, 2001.

KANEKO, J.J. (Ed.). Clinical biochemistry of domestic animals. San Diego: Academic Press, 2008. 916p.

KOTERBA, A.M.; DRUMMOND, W.H.; KOSCH, P.C. (Eds.). Equine clinical neonatology. Philadelphia: Lea \& Febiger, 1990. 846p.

LIGGINS, G.C. Premature delivery of foetal lambs infused with glucocorticoids. J. Endocrinol., v.45. p.515-523, 1969.

MARCHINI, G.; BERGGREN, V.; DJILALIMERZOUG, R.; HANSSON, L.O. The birth process initiates an acute phase reaction in the fetus-newborn infant. Acta Paediatr., v.89, p.1082-1086, 2000.

MENEGUEL, J.F.; GUINSBURG, R.; MIYOSHI, M.H. et al. Antenatal treatment with corticosteroids for preterm neonates: impact on the incidence of respiratory distress syndrome and intra-hospital mortality. Sao Paulo Med. J., v.121, p.45-52, 2003.

MILLAR, H.R.; SIMPSON, J.G., SRALKEN, A.L. An evaluation of the heat precipitation method for plasma fibrinogen estimation. J. Clin. Pathol., v.24, p.827-830, 1971 .

MOBINI, S.; HEATH, A.M.; PUGH, D.G. Teriogenologia de ovinos e caprinos. In: PUGH, D.G. (Ed.). Clínica de ovinos e caprinos. São Paulo: Roca, 2004. p.145-208.]

NOWAK, R. Neonatal survival: contributions from behavioural studies in sheep. Appl. Anim. Behav. Sci., v.49, p.61-72, 1996

PROBO, M.; GIORDANO, A.; MORETTI, P. et al. Mode of delivery is associated with different hematological profiles in the newborn calf. Theriogenology, v.77, p.865-872, 2012.
RADOSTITS, O.M.; GAY, C.C.; BLOOD, D.C. et al. Doenças do recém-nascido. In: __ Clínica veterinária: Um tratado de doenças dos bovinos, ovinos, suínos, caprinos e equinos. Rio de Janeiro: Guanabara koogan, 2002. p.102-136.

REDÜKO, S.; PRZEPIESC, J.; ZAK, J. et al. Influence of perinatal factors on hematological variables in umbilical cord blood. J. Perinat. Med., v.33, p.42-45, 2005.

STATISTICAL analysis system. Version 9.2. Cary: SAS Institute, 2008.

STEINBORN, A.; SOHN, C.; SAYEHLI, C. et al. Spontaneous labour at term is associated with fetal monocyte activation. Clin. Exp. Immunol., v.117, p.147-152, 1999.

STULL, C.; REYNOLDS, J. Calf welfare. Vet. Clin. Food Anim., v.24, p.191-203, 2008.

SZENCI, O.; TAVERNE, M.A.; TAKÁCS, E. A review of 126 caesarean sections by blood gas and the acid-base status of newborn calves. Theriogenology, v.32, p.667-674, 1989.

THILAGANATHAN, B.; MEHER-HOMJI, N.; NICOLAIDES, K.H. Labor: an immunologically beneficial process for the neonate. Am. J. Obstet. Gynecol., v.171, p.1271-1272, 1994.

TIBARY, A.; VAN METRE, D. Surgery of the sheep and goat reproductive system and urinary tract. In: FUBINI, S.L.; DUCHARME, N.G. Farm animal surgery. St. Louis: Saunders, 2004. p.527-547.

VAALA, W.E. Peripartum asphyxia. Vet. Clin. N. Am. Equine Pract., v.10, p.187-218, 1994.

VAALA, W.E.; HOUSE, J.K. Adaptação, asfixia e reanimação perinatais. In: SMITH, B.P. (Ed.). Medicina interna de grandes animais. São Paulo: Manole, 2006. p.266-276.

ZAR, J.H. Biostatistical analysis. New Jersey: Prentice-Hall, 1998. 930p.

ZOLLER, D.K.; VASSILIADISA, P.M.; VOIGTA, K.C. et al. Two treatment protocols for induction of preterm parturitionin ewes: evaluation of the effects on lung maturation and lamb survival. Small Ruminant Res., v.124, p.112-119, 2015. 